\title{
Rotary Triboelectric Nanogenerators as a Wind Energy Harvester
}

\author{
Aditya Pandey, Pushkal Badoniya, Josy George
}

\begin{abstract}
The present scenario of the world reflects the problem of energy crisis it is facing today. Increasing emissions of harmful gases like various byproducts produced by burning fossil fuels and other flue gases are adding to the worsening environmental conditions. Hence, the need of replenishable and non-conventional sources of energy is more than ever before. TENG or triboelectric nanogenerators show great potential and can be at the frontier of renewable energy technologies in the near future. Among the many applications of TENG as a harvester of abundant environmental energy, the Rotary TENG or the R-TENG is a fascinating newly found type of the same. Rotary TENG has the ability of utilizing the energy present in abundant amount in the environment, like wind energy available in the normal environment of living. Wind speed can also be measured with the help of rotary TENG when used as a self powered sensing elements. This growing field of nanotechnology can be further used in the daily lives of people in coming days.
\end{abstract}

Keywords : Nanotechnology, Rotary TENG, Self-powered sensing elements, Triboelectric nanogenerators.

\section{INTRODUCTION}

$\mathrm{T}$ he development of compact as well as smart electronics has gained pace in the current era of technology. At the same time the demand of clean and replenishable energy sources is more than ever before. The depletion of fossil fuels and the emission of harmful gases are giving rise to problems like increased percentage of carbon footprints all around the globe. The sudden rise of nanogenerators (NGs) since 2006 [1]-[5] followed by the creation of nanogenerators working on triboelectricity in 2012 [6], [7] has opened a whole new pathway for clean and renewable energy harvesting. The triboelectric nanogenerators have been tested successfully for a variety of purposes, since their emergence, by researchers around the world and they have shown great potential. A fascinating application of TENG has been tested as a device for exploiting wind energy and the TENG designed for this purpose is being called R-TENG or the rotary triboelectric nanogenerator [8].

Revised Manuscript Received on July 22, 2019.

Aditya Pandey,Mechanical department, L.N.C.T.S, Bhopal, India Pushkal Badoniya, Mechanical department, L.N.C.T.E, Bhopal, India. Josy George, Mechanical department, L.N.C.T, Bhopal, India.

\section{NON-CONVENTIONAL ENERGY SOURCES}

Non conventional energy sources are in popular use and are source of clean energy production. Renewable energy can be described as the energy that is obtained from a type of source that never outruns or exhausts its capacity. Figure 1 shows the commonly used sources of renewable energy like, wind, solar, biomass, geothermal, etc. which are derived from natural sources who get replenished in a particular amount of time. [11].

\section{A. Wind energy}

Commonly called wind power, wind energy is generated by exploiting the kinetic energy of the wind flow. Several ways have been used since a long period of time by mankind to create electricity from the flow of wind. The most traditional way of harvesting wind energy is the utilization via wind turbines [9]. The process is simple and does not requires large number of resources. The wind passes through the rotor blades and rotates them. The blades are connected to a main shaft which is in turn rotated by the rotation of the blades. This shaft rotation can be further utilized to power a generator and produce electricity. According to a report put forward by Greenpeace and the Global Wind Energy Council, by the year 2030 , wind energy could be contributing $19 \%$ of the world's overall electricity consumption. This will lead to reduction in $\mathrm{CO}_{2}$ emmisions by three billion tonnes a year, which can be a considerable support to the other renewable sources like solar energy and biomass, just to name a few. Furthermore, by the year 2050, wind energy could be powering $25-30 \%$ of the cumulative energy expenditure of the world [10]. The major advantages of considering wind energy as a better renewable energy source are its ability to be widely distributed, no water consumption while the operation and little land coverage.

\section{B. Solar Energy}

Other than the day to day application of solar energy for lighting purposes in households, it is utilized by two distinct methods namely photovoltaic (PV) technology and solar thermal. The first method of Photovoltaic utilization is the exploitation of solar cells for conversion of radiation from the sun directly into electric power. When the PV cells are put together a PV panel is obtained. Furthermore, the approach of Solar thermal (ST) is the technology which applies solar energy extracted from the sun to make thermal energy [12]. 
Solar energy is an applaudable natural energy source. Some drawbacks in these particular methods include its costly setup and the fact that sunlight can be harvested only during daytime. Other than that solar power can't also be utilized in the season of rainfall and cloudy weather conditions.

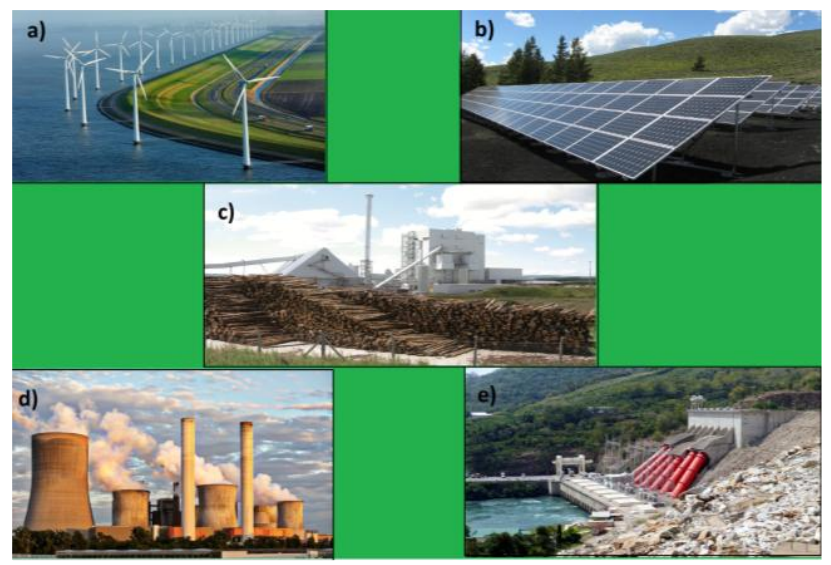

Fig. 1. Various sources of renewable energy: a) A wind-mill setup. b) A solar farm. c) Biomass conversion plant. d) Geothermal power plant. e) Hydroelectric power plant.

\section{Geothermal Energy}

Geothermal is made up of greek words 'geo' which means earth and 'thermal' meaning heat. This derived definition points towards the actual meaning of geothermal which is heat generated from inner surface of the earth. Due to radioactive decay phenomenon beneath the surface of earth geothermal energy is generated and trapped inside the surface. An important reason of the radioactive decaying is the high temperatures (about 4000 degrees) found deep inside the earth's surface. The energy available at these depths are exploited with the help of various technologies available today. Frequent use of a key factor known as geothermal gradient is commonly seen for determining the areal enactment. It works as a relative scale by relating the depth and earth's temperature which are directly proportional to each other. Therefore, active volcanic areas are best suited for construction of geothermal power stations according to geothermal gradient. These plants are costly to construct initially but further processing and other operational costs are comparatively less. A major disadvantage is considered to be the surface instability in the area where plant is located and this can lead to phenomena like earthquakes.

\section{Hydro Power}

Running water is used to obtain energy called hydro power. It can be said that it is the conversion of energy of flowing water directly into electricity which can be utilized for many necessary purposes. Hydro power is better than wind power because of the fact that water has higher density than air resulting in more energy generation. The fact that resulting efficiency can be modified as per the required value by restricting the running water movement is an important and considerable advantage associated with hydro power. Currently, because of excessive commencing charges and various rules and regulations regarding environmental safety, the installation of a plant is strenuous. Itaipu Dam installed on the Parana river has the largest capacity in the world. China being leading manufacturer of hydroelectricity has corresponding electricity generation being 694.0 TWh [14].

\section{E. Biomass}

Biomass is actually the residual byproducts or unusable matter derived from vegetation and waste from dead or alive organisms. Biomass is famously processed for generation of fuel which in-turn is used for energy production. Since the ancient times, biomass is being used by mankind in many ways, mainly for feedstock and firewood purposes. Easy access to biomass is a considerable advantage due to which it is readily used. Farming of energy crops is practised across the country and it is used for biofuel manufacturing. Major processes involved in biomass processing are pyrolysis and combustion which come under thermo-chemical treatment and anaerobic digestion which comes under bio-chemical treatment, either one of which is given priority depending upon the type of fuel required. In case of emmisions, biomass is way better than conventional energy sources, which emit large amount of harmful gases as residual products. Furthermore, bio-crops are cultivated for conversion into biofuels by thermal treatment, in order to be used as an alternative or additive to diesel [15].

\section{F. Triboelectric Nanogenerators (TENG)}

The methods of generating renewable energy discussed above have associated drawbacks and limitations which affects their application and large-scale use. Demand of clean energy sources has been increased in recent times, and emerging technologies like biofuel manufactured from cellulosic ethanol and power developed from marine energy are in the development phase which will help in fulfilling the demand of energy supply around the globe in the upcoming future [16] Triboelectric nanogenerators, after their discovery, have opened a new branch in renewable sources of energy harvesting which sheds light on potential hybridized nano-technologies capable of clean energy generation. Various praiseworthy designs and fabricated models have been demonstrated derived from the basic TENG concept, to harvest different forms of abundant energy available in the environment. Figure 2 shows the different designs like biodegradable triboelectric nanogenerator (BD-TENG) [17], paper-based TENG for harvesting acoustic energy [18], TENG as an electronic skin for harvesting biomechanical energy [19] and TENG driven by elasto-aerodynamics for utilizing energy from flow of air [20] which are promising and have the potential to provide clean energy at small as well as large scale. Moreover, TENG have also been utilized as self-powered sensors in technologies like non-contact free rotating TENG utilized as self-powered mechanical sensor [21] and TENG matrix for instantaneous tactile imaging [22] only to name a few. 


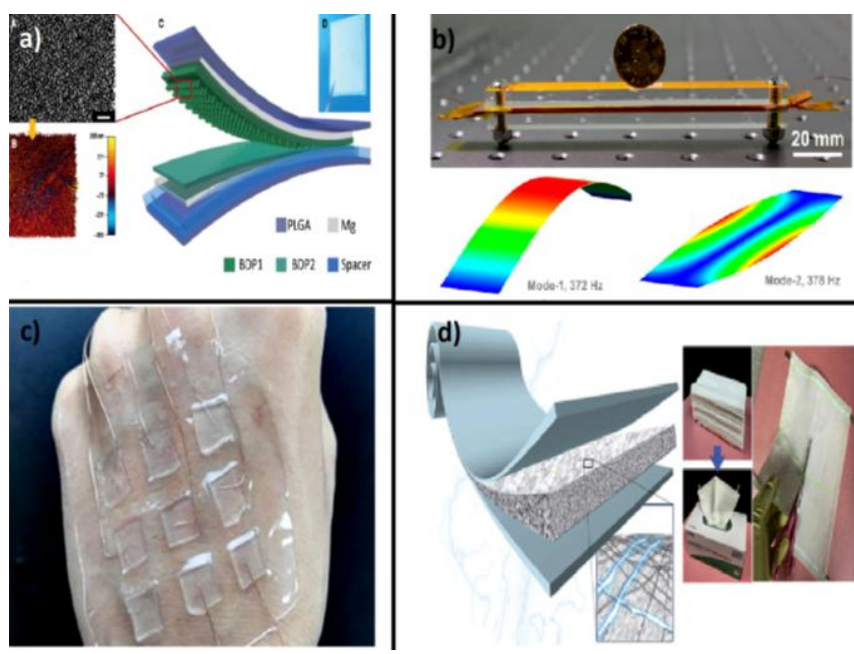

Fig. 2. Different designs of triboelectric nanogenerator : (a) Bio-degradable TENG [17]. (b) Elasto-aerodynamics driven TENG [20]. (c) Transparent TENG for tactile sensing [19]. (d) Paper-based TENG [18]

\section{1) Rotary Triboelectric Nanogenrators (R-TENG)}

In 2012, the first model of triboelectric nanogenerator (TENG) was demonstrated which worked basically on the combined principle of triboelectric effect, which is generally considered a negative phenomenon in industries due to its tendency of static discharge which can cause dust explosion, ignition and damage to electronic devices. The fabrication of TENG does not require enough resources and uses only conventional materials for manufacturing. The basic principle behind the functioning of triboelectric nanogenerator requires the simultaneous contacting and spliting between two oppositely tribo-charged surfaces having opposite polarity. Change in the electrodes' induced potential difference is simultaneously observed and hence an alternated electron flow is generated [23-25]. Figure 3 shows the recently developed rotary type TENG which uses basically the same principle but it utilizes the cyclic procedure of contact-sliding-seperation-contact to create the electron flow across the load. This new advancement in TENG technology is useful in extracting small scale wind energy present in the environment. The wind is used to rotate the cup structures which in turn rotates the PTFE thin films across the stationary aluminium films and generates the contact-sliding-seperation-contact cyclic process which continues until the wind flow takes place.

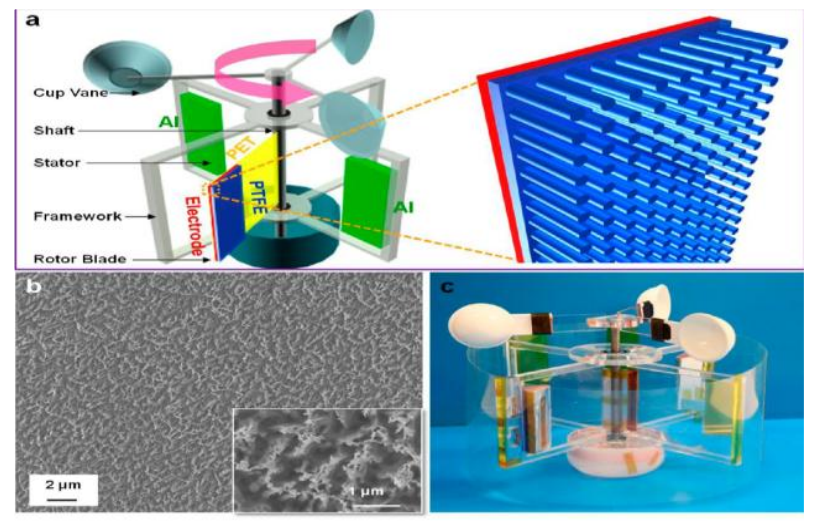

Fig. 3. Rotary TENG: (a) Structure of R-TENG along with a large-scale view of the nanowire like extensions on the PTFE outer surface. (b) Image obtained from SEM analysis of PTFE surface with an inset SEM image of nanowire structures. (c) A fabricated model of R-TENG device [8].

\section{PROCEDURE}

The rotary TENG consists of a framework which supports the rotor blade, the stator, wind cup structures and the shaft at the centre. Shaft is held with the support of bearings at both ends. Rotor is directly connected to the shaft for simultaneous rotary motion. The working mechanism is basically divided into two parts namely the sliding mode [26]-[28] and the contact separation mode [23]-[25]. Both these modes take place one after the other and hence complete a cycle to generate a pair of alternating currents.

\section{A. Sliding Mode}

As depicted by figure $4((a)(b)(c))$, initially the rotor is stationary and it is separated from the other triboelectric layer. Now, the wind is allowed to flow and the cups start to rotate and they, in turn, rotates the rotor blade. Full contact between the blade and either one of the aluminium stators is observed. Due to relative sliding which takes place between PTFE and $\mathrm{Al}$, opposite charges are generated on both surfaces [23, 24, $31,32]$. As the rotation action continues the rotor blade with PTFE film slides outward across the adjacent surface of aluminium, therefore a decrement in the overlapping area takes place. Owing to this, induction of higher potential takes place on the aluminium surface, which in turn is responsible for flow of current across the load to the PTFE electrode. As the sliding effect creates more friction as compared to the contact separation mode, it is more capable of generating charge densities. In order to reduce the bending action generated during the sliding takes place, the PTFE film is joined with a PET (Polyethylene Teraphthalate) film. This PET film is subjected the most part of bending action while the sliding action is consumed by the adjacent PTFE film.

\section{B. Contact-separation mode}

Figure 4.((d),(e)) shows that after the completion of sliding mode working mechanism, the rotor blade moves forward with the same amount of opposite charge densities over PTFE film and meets the other Al stator momentarily for vertical contact [33]. The reversed flow of current now takes place from the PTFE surface to the Al film on the stator, hence transferring the positive charge densities to the $\mathrm{Al}$ sheet and to reach a state of equilibrium. This completes a cycle of rotation of rotor blade which utilized the hybridized eparation-contacting-sliding-separation concept. The whole process generates alternating current at two points of contact and recontact with $\mathrm{Al}$ stator sheet. During the first contact correlating with the in plane separation of charges, a current with lower magnitude and a comparatively broader tip is observed. Whereas, during the vertical recontact, an alternating current with smaller width, but comparatively acute tip is detected.

\section{Output Observations}

As the whole process was being governed by the wind speed, it was fixed to an approximate value of $15 \mathrm{~m} / \mathrm{s}$. As per the observations gathered by the experimentalists, the output open circuit voltage reached a maximum value of $250 \mathrm{~V}$ along with the short circuit current value reaching $0.25 \mathrm{~mA}$. Also, the maximum power obtained in the experiment was noted to be $62.5 \mathrm{~mW}$, corresponding to the fixed wind speed [8]. 


\section{Factors Affecting Performance of R-TENG}

As discussed above, the outputs were measured by keeping the wind speed constant, due to the fact that the output values vary with changing wind speed. Other factors which affect the working of the rotary-type triboelectric nanogenerators include the material selection and the framework used for assembly. These factors govern the performance of the rotary TENG and play a dominating part in deciding the overall efficiency of the same.

\section{A. Material Selection}

Selection of material is required for sheets on both the stator and the rotor blade. During the selection of material, it should be kept in mind that both the materials must be capable of acquiring opposite charges when they are made to come in contact with each other and it must be lightweight. The fabricated R-TENG consists of PTFE material adhered to the layer of PET assembled on the rotor blade, due to its property of acquiring negative charge when made to come in contact with a material present on the upper half of triboelectric series [29]. Aluminium sheet is used on the stationary stator because aluminium has the tendency to acquire positive charge when touched with a material below it in the triboelectric series. Hence, PTFE and Al sheets form a combination of opposite charge acquiring materials suitable for R-TENG. As for the electrodes, the first one is present in the form of $\mathrm{Al}$ sheet which acts as a material according to the triboelectric series as well as an electrode, and for the second one, a thin film of metal is embedded between the polytetrafluoroethylene and polyethylene terephthalate films present on the rotor blade. Surface roughness also plays a major role in improving the charge density of materials. Etching procedure is used on the PTFE film in order to create constantly distributed nanowire-like structures on its surface [30].

\section{B. Framework}

A proper framing of the R-TENG is necessary for utilizing most of the wind energy. The demonstrated design shows the shaft at central axis which is supported by two bearings. Two rectangular acrylic frames are joined at right angles to each other at the central axis on which the shaft is placed. A rotor and two stators are also included in the design. Rotor is directly attached to the shaft for simultaneous rotation. Wind cup-like structures are mounted over the shaft for converting the wind into rotary motion. Another important aspect is the selection of accurate number of stators to be used in R-TENG for effective generation of electricity. R-TENG devices with three stators and also with four stators were fabricated with similar design for comparison of resulting output with the R-TENG having two stators. After equating the output produced, it was found that maximum and most balanced output was generated by using the design consisting of two stators. The explanation behind could be the fact that, when the number of stators is increased, the space available between two consecutive stators is less and due to this the rotor couldn't recover its original shape, hence the decrement in face to face contact takes place which decreases the efficiency.

\section{Wind Speed}

On investigation, it was found that a close relationship takes place between the electrical production of R-TENG and the wind velocity. Similar experiments were demonstrated with two different wind speeds and it was observed that when the speed is increased the open circuit voltage showed a little increment and then reached its capacity of $250 \mathrm{~V}$ when the force of contact acquired highest value [7], [23], [25]. The short circuit current increased with elevating the wind speed due to increment in charge transfer rate, thus power output of the R-TENG also showed considerable raise.

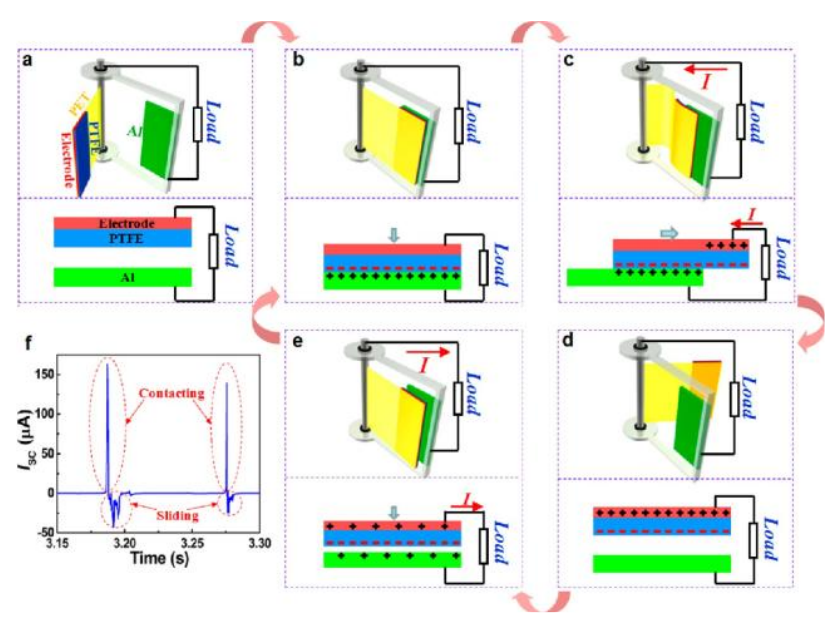

Fig. 4. Procedure involved in R-TENG: (a), (b), (c) Sliding mode. (d), (e) Contact separation mode. (f) Current output profile [8].

\section{OTHER DEVICES FOR ROTARY MOTION HARVESTING}

Other than the R-TENG device discussed above, other innovative designs have been demonstrated by researchers. Hybrid processes have been applied by combining various contact modes of TENG in order to develop new durable designs with higher efficiencies.

\section{A. Self Powered Wind Speed Sensors}

The application of R-TENG as a device capable of harvesting wind energy has already been discussed above. The same approach has been applied to acquire information about the wind speed. Figure 5.(a),(b) shows the graph relating the $I_{\mathrm{sc}}$ and $\mathrm{V}_{\mathrm{oc}}$ to the time, used to determine the real-time wind speed. Time gap between two consecutive spikes or the number of spikes obtained in a certain time interval can be used to determine the wind speed. This phenomenon can be described as due to the increment in wind speed the rotor rotates faster which in-turn increases the rate of output occurrence. Hence, the wind velocity associated with the peaks obtained in a certain time interval can be determined and this demonstrates the use of working principle applied in the rotary triboelectric nanogenerator, utilized as a self-powering active sensing element [8], [36].

\section{B. TENG Associated With Air Cleaning Technology}

Utilizing the advantage of high output produced in rotary triboelectric nanogenerator with grating electrode, an air cleaning system has been fabricated which uses natural flow of wind in order to remove sulphur dioxide $\left(\mathrm{SO}_{2}\right)$ and particulate matters (PMs) from the air.

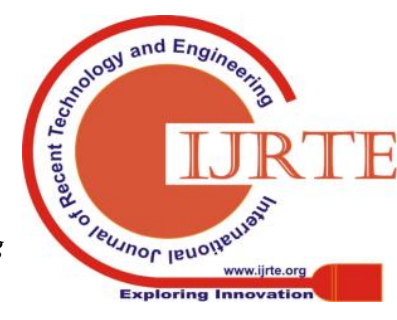


Electrostatic force is used to charge the polyimidic (PI) nanofibres for the successful removal of particulate matter. $\mathrm{SO}_{2}$ oxidation is required in order to remove it from atmosphere. Figure 5.(c) shows the designed structure in which, with the help of R-TENG connected to a rectifying bridge, alternating current developed in the R-TENG is converted to DC current. The rectifying bridge is connected to a chamber which is initially filled with $\mathrm{SO}_{2}$ gas and contains an anode and a cathode. $\mathrm{NaHSO}_{3}$ solution is kept in the chamber due to the fact that sulphur dioxide is not soluble in this solution. A film of evaporated $\mathrm{NaHSO}_{3}$ is formed on the copper mesh, which makes the oxidation possible. It was found that the wind speed had a direct relation with concentration of $\mathrm{SO}_{2}$. As the wind speed was increased, concentration of $\mathrm{SO}_{2}$ decreased rapidly. The reason behind the decrement in rate of concentration of sulphur dioxide can be associated with the current density at the anode, which is responsible for the rapid oxidation process. This current density at the anode, in-turn, depends on the wind speed, hence describing this phenomenon [35].

\section{Rotary Sliding Disk TENG}

RD-TENG is a new and emerging design which exists in two forms, namely, dielectric RD-TENG and metal RD-TENG. Both of these types work on contact mode and non-contact mode. FEM (finite element method) analysis showed that the characteristics and execution of RD-TENG is controlled highly by the material selection. PTFE and aluminium were chosen in the designing of RD-TENG, which are suitable triboelectric materials. Electricity is generated by in-plane charge density separation corresponding to the rotation of disks. The calculations performed on FEM were based on some parameters which governed the output, namely, dielectric constants, inside and outside radius' of triboelectric nanogenerator, tribo-charge surface density, grating number,center angle corresponding to grating part and angular rotational velocity [34]. A practically fabricated model is still lacking as the design is in the initial stages, but the approach has shown potential and work is being done on the concept. Figure 5.(d) shows the proposed concept design of RD-TENG.

\section{CHALLENGES}

TENG as a developing technology is promising and is capable of leading the renewable energy market in the coming days. Various hybridization techniques have been put forward by researchers which show potential and are able to produce efficient output. Even though the progress of these technologies has gained momentum, some challenges are needed to be overcome and some concepts are yet to be studied thoroughly. R-TENG devices confront some issues which rose questions that are needed to be addressed.

\section{A. Fundamentals of Tribolectric Effect}

The physics associated with triboelectrification requires deep and thorough study. Progress has been made in this field, but it still remains as a concept yet to be understood completely. Research is currently in progress and microscopy techniques are being adopted for this purpose. It is required to commence related calculations from the basic principles to better understand the triboelectrification phenomenon. Atomic force microscopy (AFM) has been applied to intentionally change the effect produced by contact electrification process through the introduction of an electric field between two materials [37]. Scanning kelvin probe microscopy (SKPM) is being used for measurement of change in original surface potential associated with the contact area [38].

\section{B. High Performance Materials}

The important element in determining the power output of TENG is surface charge density. Hence, a material with high surface charge density is required. Moreover, the dielectric constant of materials accounts for the electrostatic induction phenomenon in the triboelectric nanogenerators. The effectiveness of the surface contact between the two materials improves the output efficiency, which is solely a function of materials used in the process. Creating hybrid materials with enhanced properties is an ongoing topic of interest for researchers. Praiseworthy advancements in material improvement have been recently demonstrated and further studies are also being done [39]- [41].

\section{Durability}

This is a major concern as the problem of decay in the TENG surface charge density could affect its output performance. The need is to design materials with high mechanical stability and robustness, so that they remain intact for a longer time period without interfering with the operation of TENG. A highly durable and stretchable TENG based on gold nanosheet-embedded electrode was subjected to successful stability tests [42]. Also, sliding friction replaced by rolling friction results in increased robustness and decreased wear and tear of the material which in-turn enhances the efficiency of the instantaneous energy conversion [43]. Composite materials are also an option which require improvements in pursuance of better durability of the design.

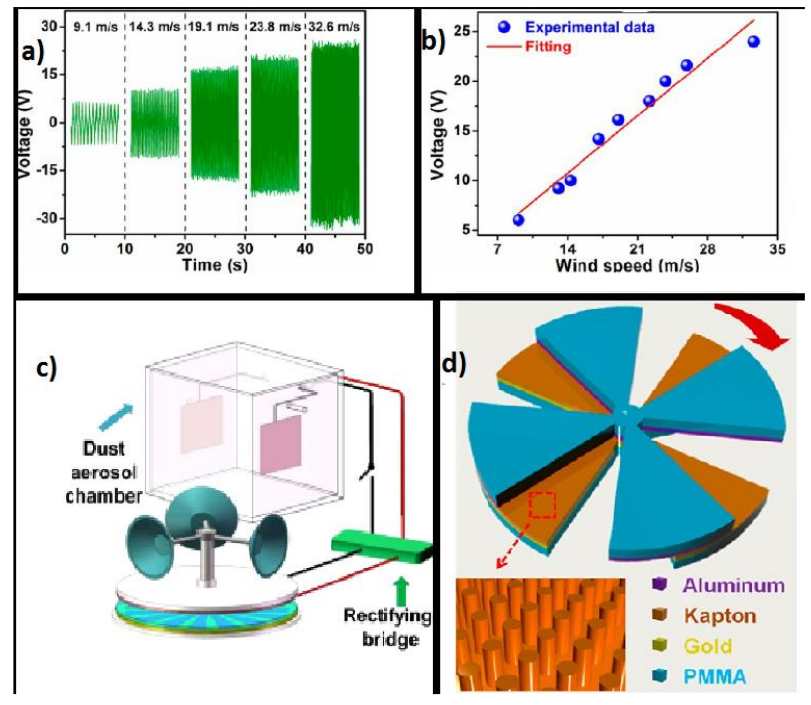

Fig. 5. Devices based on rotary motion harvesting: (a) Relation between output voltage and wind speed. (b) Relation between voltage and wind speed shows a linear trend [46]. (c) Self-powered SO2 oxidation driven by R-TENG [35]. (d) Structure of cylindrical rotating TENG [47]. 


\section{Environmental Effects}

Previous works have shown that TENG works better in a vacuum condition due to increment in charge density by a factor of 5 [44]. Thus, it is necessary to create better packing materials so that a quasi-vacuum condition is attained in the system. Also, the temperature limits for effective operation of TENG have been previously determined to be from $-196.1^{\circ} \mathrm{C}$ to about $400^{\circ} \mathrm{C}$ [45], but further increasing these limits still remains a limitation for concerned researchers. Hence, tasks like prevention from environmental effects like contact with water and interference of moisture and atmosphere with the system is necessary as these factors highly control the device's efficiency.

\section{CONCLUSION}

In a summarized way, it is clear that since the invention of triboelectric nanogenerator, development in the relative technology and principles has taken place. These developments have opened several different branches which are based on the modified principles and use hybridized processes to achieve different objectives. A similar branch of TENG known as rotary TENG has been reviewed in this paper which focuses on rotary motion in order to harness the wind energy. The demonstrations have shown that the R-TENG device has great potential in harvesting natural wind, especially the weak wind, present in abundant amount in the environment. The initial experiments showed that the output obtained were promising with the voltage $\left(\mathrm{V}_{\mathrm{oc}}\right)$ and current $\left(\mathrm{I}_{\mathrm{sc}}\right)$ being 250 Volts and $0.25 \mathrm{~mA}$ respectively with the wind speed being fixed at $15 \mathrm{~m} / \mathrm{s}$. Moreover, the same device has been demonstrated to be used as real time wind speed sensors for determining wind speed. This indicates the ability of this technology to lead the wind power technology in the near future. The demand of similar technology is more than ever due to the various drawbacks and limitations associated with the conventional wind turbines which are commonly used for harnessing wind power. Various other similar technologies working on the rotary motion have been developed which were discussed above. The rotary-sliding disk TENG (RD-TENG) is still in the development stage but the demonstrations performed in finite element method analysis have shown successful operation. The use of R-TENG for development of air cleaning system to remove $\mathrm{PMs}$ and $\mathrm{SO}_{2}$ from the air is an innovative concept which has been realized already. The combination of triboelectric nanogenerators with the conventional wind energy technologies, has shown a number of advantages. In the coming days, with the advancement in development of hybrid materials and continuous work by researchers across the globe, the performance of TENG will only increase and soon this nano-technology will upgrade and provide ease to the human living.

\section{REFERENCES}

1. Z. L. Wang, "Piezoelectric Nanogenerators Based on Zinc Oxide Nanowire Arrays," in Science, vol. 312, no. 5771, 2006, pp. 242-246.

2. X. Wang, J. Song, J. Liu, and Z. L. Wang, "Direct-Current Nanogenerator Driven by Ultrasonic Waves," in Science, vol. 316, no. 5821, 2007, pp. 102-105.

3. Y. Qin, X. Wang, and Z. L. Wang, "Microfibre-nanowire hybrid structure for energy scavenging," in Nature, vol. 451, no. 7180, 2008, pp. 809-813.
4. R. Yang, Y. Qin, L. Dai, and Z. L. Wang, "Power generation with laterally packaged piezoelectric fine wires," in Nature Nanotechnology, vol. 4, no. 1, 2008, pp. 34-39.

5. S. $\mathrm{Xu}$ et al., "Self-powered nanowire devices," in Nature Nanotechnology, vol. 5, no. 5, 2010, pp. 366-373.

6. F.-R. Fan, Z.-Q. Tian, and Z. L. Wang, "Flexible triboelectric generator," in Nano Energy, vol. 1, no. 2, 2012, pp. 328-334.

7. F.-R. Fan et al., "Transparent Triboelectric Nanogenerators and Self-Powered Pressure Sensors Based on Micropatterned Plastic Films," in Nano Letters, vol. 12, no. 6, 2012, pp. 3109-3114.

8. Y. Xie et al., "Rotary Triboelectric Nanogenerator Based on a Hybridized Mechanism for Harvesting Wind Energy," in ACS Nano, vol. 7, no. 8, 2013, pp. 7119-7125.

9. Bressers, S. et al., "Small-scale modular windmill," in American Ceramic Society Bulletin. vol. 89, no. 8, 2010, pp. 34-40.

10. L. Fried, S. Sawyer, S. Shukla and L. Qiao, "Global Wind Energy Outlook", in Global Wind Energy Council, Brussels, 2014.

11. O. Ellabban, H. Abu-Rub, and F. Blaabjerg, "Renewable energy resources: Current status, future prospects and their enabling technology," in Renewable and Sustainable Energy Reviews, vol. 39, 2014, pp. 748-764.

12. B. Norton, "Harnessing Solar Heat," in Lecture Notes in Energy, 2014.

13. I. Fridleifsson et al.: "The possible role and contribution of geothermal energy to the mitigation of climate change", in IPCC Scoping Meeting on Renewable Energy Sources. Intergovernmental Panel on Climate Change, pp. 59 - 80, 2008, Switzerland.

14. D. Magee, "Hydropower and End-Use Electrical Efficiency in China: State Support and Potential Contribution to Low-Carbon Development," in The Copenhagen Journal of Asian Studies, vol. 33 no. 1,2015 , p. 64

15. A. Kumar, N. Kumar, P. Baredar, and A. Shukla, "A review on biomass energy resources, potential, conversion and policy in India," in Renewable and Sustainable Energy Reviews, vol. 45, 2015, pp. 530-539.

16. "Renewables in Global Energy Supply: An IEA facts sheet", 2007.

17. Q. Zheng et al., "Biodegradable triboelectric nanogenerator as a life-time designed implantable power source," in Science Advances, vol. 2 , no. 3, 2016

18. X. Fan et al., "Ultrathin, Rollable, Paper-Based Triboelectric Nanogenerator for Acoustic Energy Harvesting and Self-Powered Sound Recording," in ACS Nano, vol. 9, no. 4, pp., 2015, 4236-4243.

19. X. Pu et al., "Ultrastretchable, transparent triboelectric nanogenerator as electronic skin for biomechanical energy harvesting and tactile sensing," in Science Advances, vol. 3, no. 5, 2017.

20. S. Wang et al.,"Elasto-Aerodynamics-Driven Triboelectric Nanogenerator for Scavenging Air-Flow Energy," in ACS Nano, vol. 9, no. 10, 2015, pp. 9554-9563.

21. L. Lin et al., "Noncontact Free-Rotating Disk Triboelectric Nanogenerator as a Sustainable Energy Harvester and Self-Powered Mechanical Sensor," in ACS Applied Materials \& Interfaces, vol. 6 , no. 4, 2014, pp. 3031-3038.

22. Y. Yang, H. Zhang, X. Zhong, F. Yi, R. Yu, Y. Zhang, and Z. L. Wang, "Electret Film-Enhanced Triboelectric Nanogenerator Matrix for Self-Powered Instantaneous Tactile Imaging," in ACS Applied Materials \& Interfaces, vol. 6, no. 5, 2014, pp. 3680-3688.

23. G. Zhu et al., "Triboelectric-Generator-Driven Pulse Electrodeposition for Micropatterning," in Nano Letters, vol. 12, no. 9 , 2012, pp. 4960-4965.

24. S. Wang, L. Lin, and Z. L. Wang, "Nanoscale Triboelectric-Effect-Enabled Energy Conversion for Sustainably Powering Portable Electronics," in Nano Letters, vol. 12, no. 12, 2012 , pp. 6339-6346.

25. G. Zhu et al., "Toward Large-Scale Energy Harvesting by a Nanoparticle-Enhanced Triboelectric Nanogenerator," in Nano Letters, vol. 13, no. 2, 2013, pp. 847-853.

26. S. Wang et al., "Sliding-Triboelectric Nanogenerators Based on In-Plane Charge-Separation Mechanism," in Nano Letters, vol. 13, no. 5, 2013, pp. 2226-2233.

27. G. Zhu et al., "Linear-Grating Triboelectric Generator Based on Sliding Electrification," in Nano Letters, vol. 13, no. 5, 2013, pp. 2282-2289.

28. L. Lin et al., "Segmentally Structured Disk Triboelectric Nanogenerator for Harvesting Rotational Mechanical Energy," in Nano Letters, vol. 13, no. 6, 2013, pp. 2916-2923. 
29. A. Diaz and R. Felix-Navarro, "A semi-quantitative tribo-electric series for polymeric materials: the influence of chemical structure and properties," in Journal of Electrostatics, vol. 62, no. 4, 2004, pp. 277-290.

30. H. Fang, W. Wu, J. Song, and Z. L. Wang, "Controlled Growth of Aligned Polymer Nanowires," in The Journal of Physical Chemistry C, vol. 113 , no. 38,2009 , pp. $16571-16574$

31. L.-H. Lee, "Dual mechanism for metal-polymer contact electrification," in Journal of Electrostatics, vol. 32, no. 1, 1994, pp. $1-29$.

32. P. K. Watson and Z.-Z. Yu, "The Contact Electrification of Polymers and the Depth of Charge Penetration," in Journal of Electrostatics, vol. 40-41, 1997, pp. 67-72.

33. F. Saurenbach, D. Wollmann, B. D. Terris, and A. F. Diaz, "Force microscopy of ion-containing polymer surfaces: morphology and charge structure," in Langmuir, vol. 8, no. 4, 1992, pp. 1199-1203.

34. T. Jiang et al., "Theoretical study on rotary-sliding disk triboelectric nanogenerators in contact and non-contact modes," in Nano Research, vol. 9 , no. 4, 2016, pp. 1057-1070.

35. C. He and Z. L. Wang, "Triboelectric nanogenerator as a new technology for effective PM2.5 removing with zero ozone emission," in Progress in Natural Science: Materials International, vol. 28, no. 2, 2018, pp. 99-112.

36. G. Zhu, J. Chen, T. Zhang, Q. Jing, and Z. L. Wang, "Radial-arrayed rotary electrification for high performance triboelectric generator," in Nature Communications, vol. 5, no. 1, 2014.

37. Y. S. Zhou et al., "Manipulating Nanoscale Contact Electrification by an Applied Electric Field," in Nano Letters, vol. 14, no. 3, 2014, pp. 1567-1572.

38. Y. S. Zhou, S. Li, S. Niu, and Z. L. Wang, "Effect of contact- and sliding-mode electrification on nanoscale charge transfer for energy harvesting," in Nano Research, vol. 9, no. 12, 2016, pp. 3705-3713.

39. Y. Dong et al., "Metallic MXenes: A new family of materials for flexible triboelectric nanogenerators," in Nano Energy, vol. 44, 2018, pp. $103-110$

40. S. S. K. Mallineni et al., "Facile and robust triboelectric nanogenerators assembled using off-the-shelf materials," in Nano Energy, vol. 35, 2017, pp. 263-270.

41. X. He et al., "A Highly Stretchable Fiber-Based Triboelectric Nanogenerator for Self-Powered Wearable Electronics," in Advanced Functional Materials, vol. 27, no. 4, 2016, p. 1604378.

42. G.-H. Lim et al., "Fully stretchable and highly durable triboelectric nanogenerators based on gold-nanosheet electrodes for self-powered human-motion detection," in Nano Energy, vol. 42, 2017, pp. 300-306

43. L. Lin et al., "Robust Triboelectric Nanogenerator Based on Rolling Electrification and Electrostatic Induction at an Instantaneous Energy Conversion Efficiency of $\sim 55 \%$," in ACS Nano, vol. 9, no. 1, 2015 , pp. 922-930.

44. J. Wang et al., "Achieving ultrahigh triboelectric charge density for efficient energy harvesting," in Nature Communications, vol. 8, no. 1, 2017.

45. C. Xu et al., "Raising the Working Temperature of a Triboelectric Nanogenerator by Quenching Down Electron Thermionic Emission in Contact-Electrification," in Advanced Materials, vol. 30, no. 38, 2018 p. 1803968.

46. H. Zhang et al., "Single-Electrode-Based Rotating Triboelectric Nanogenerator for Harvesting Energy from Tires," in ACS Nano, vol 8 , no. 1,2013 , pp. 680-689.

47. P. Bai et al., "Cylindrical Rotating Triboelectric Nanogenerator," in ACS Nano, vol. 7, no. 7, 2013, pp. 6361-6366.

\section{AUTHORS PROFILE}

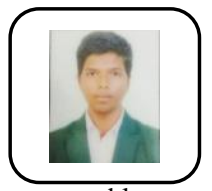

Mr. Aditya Pandey is currently pursuing his Bachelor's degree in Mechanical Engineering from RGPV University. He has published and presented a couple of papers in international conferences held in reputed universities. His main research interest includes renewable energy resources, nanotechnology and nanogenerators. He looks forward to pursue higher studies in the field of renewables and nanogenerators. The conferences include International Conference on ATREFS-2019 organised by School of Energy \& Environment Management, Rajiv Gandhi Proudyogiki Vishwavidyalaya; International Conference on Interdisciplinary Research in Engineering, Pharmaceutical \& Applied Sciences (ICIREMPS 2K18-19) held at Sagar Group of Institutions,
Bhopal and $1^{\text {st }}$ International Conference on Clean and Renewable Energy (ICCARE 2019) held at NIT Durgapur.

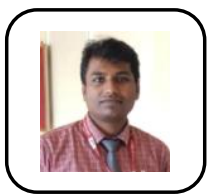

Mr. Pushkal Badoniya is an Assistant Professor in Mechanical Engineering Department in Lakshmi Narain College of Technology Excellence, Bhopal, India. He completed his Bachelor's degree course in the field of Mechanical Engineering from RGPV University, Bhopal. He obtained his Master's Degree in Manufacturing Technology from NIT Jalandhar. He has over 5 years of teaching experience. His main research interest includes renewable energy sources, Industrial and production engineering. He has published or presented various research papers in reputed National/International Journal/Conferences. He has also written a book titled "Solar Air Heater: Different Approaches to the Investigation". He is expert in ANSYS, ProE, AutoCAD, SigmaPlot, MATLAB, and LaTex.

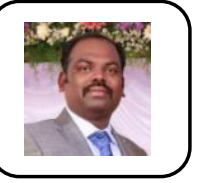

Mr. Josy George is an Assistant Professor in Mechanical Engineering Department in Lakshmi Narain College of Technology, Bhopal, India. He received his Bachelor's Degree in Mechanical Engineering from Rajiv Gandhi Proudyogiki Vishwavidyalaya (RGPV), Bhopal, Madhya Pradesh, India. He obtained his Master's Degree in Industrial Engineering and Management from Institute of Engineering \& Technology (IET-DAVV). IET-DAVV is an Engineering Branch of Devi Ahilya Vishwavidyalaya, Indore, Madhya Pradesh, India. He has over 8 years of teaching experience and 2 years of industrial experience. $\mathrm{He}$ has published or presented various research papers in reputed National/International Journal/Conferences. He has also written a book titled "Solar Air Heater: Different Approaches to the Investigation". He is expert in SolidWorks and AutoCAD. 